\title{
Dezentrale optimale Betriebsstrategie für Hybridantriebe
}

\author{
Dipl.-Ing. Alexander Frenkel, Prof. Dr.-Ing. Rainer Bruns \\ Helmut-Schmidt-Universität Hamburg \\ Lehrstuhl für Maschinenelemente und Technische Logistik
}

\begin{abstract}
Der vorliegende Beitrag befasst sich mit einer dezentralen und optimalen Regelung von Hybridantrieben. Die Methode wird vorgestellt und in ihren wesentlichen Punkten hergeleitet. Am Beispiel eines parallelen Hybridantriebes mit Momentaddition für Flurförderzeuge wird die Methode angewandt. Zur Beurteilung der Güte wird für den gewählten Hybridantrieb und ein standardisiertes Fahrprofil der optimale Betrieb des Antriebstranges mit Hilfe der dynamischen Programmierung ermittelt. Im Vergleich dieser optimalen Lösung mit der dezentralen Betriebsstrategie zeigt sich eine sehr gute Übereinstimmung.
\end{abstract}

\section{$1 \quad$ Einleitung}

Der vorliegende Beitrag stellt eine Methode zur optimalen dezentralen Regelung von Hybridantrieben vor. Hybridantriebe stellen verteilte Energieflusssysteme dar, welche einen oder mehrere interne Freiheitsgrade aufweisen. Regelungen und Steuerungen solcher verteilter Systeme nehmen unter einer Optimalitätsforderung schnell sehr komplexe Formen an, wenn a priori zu jedem Zeitpunkt, für jeden Systemzustand und jede denkbare Fehlfunktion einzelner Subsysteme eine adäquate Systemreaktion implementiert werden soll. Hinzu kommt, dass eine Systemänderung eine äußerst aufwendige Anpassung der Regelung nach sich zieht. Aus diesem Grund werden die Steuerungen unter Verzicht auf strenge Optimalität häufig dezentral ausgelegt. Ausgezeichnete Subsysteme erhalten in diesem Fall eine lokale Steuerung. Durch Kommunikation untereinander und geschickter Auslegung der dezentralen Regeln kann ein emergentes Systemverhalten mit hinreichenden Eigenschaften bezüglich Optimalität, Robustheit und Flexibilität unter vertretbarem Aufwand implementiert werden.

Die im Folgenden vorgestellte und diskutierte Methode zur dezentralen Regelung wurde am konkreten Beispiel Hybridantrieb für Gabelstapler entwickelt, lässt sich aber prinzipiell auch auf andere geregelte und verteilte Netzwerke übertragen. Wie gezeigt werden wird, strebt das System trotz dezentraler Regelung zu jedem Zeitpunkt gegen den Zustand eines global definierten Optimums. Dabei geht in die Auslegung des lokalen Reglers nur Systeminformation der zu regelnden Instanz ein. Diese Vorgehensweise birgt den Vorteil, dass das Gesamtsystem verändert werden kann, ohne die Betriebsstrategie neu auslegen zu müssen. 


\section{Grundlagen Hybridantriebe}

\subsection{Antriebsstrang}

Hybridantriebe für Fahrzeuge bestehen definitionsgemäß aus mindestens zwei unterschiedlichen Einzelantrieben und zwei unterschiedlichen Energiespeichern [UN03]. Die Motivation zum Einsatz derartiger komplexer und teurer Systeme gründet sich überwiegend in der Betriebskostenersparnis, die durch die Kombination der Stärken zweier verschiedener Antriebe erwartet wird. Die eingesetzten Einzelantriebe müssen daher so miteinander kombiniert werden, dass der Energieverbrauch des Fahrzeugs gegenüber einem Vergleichbaren mit konventionellem Antrieb sinkt.
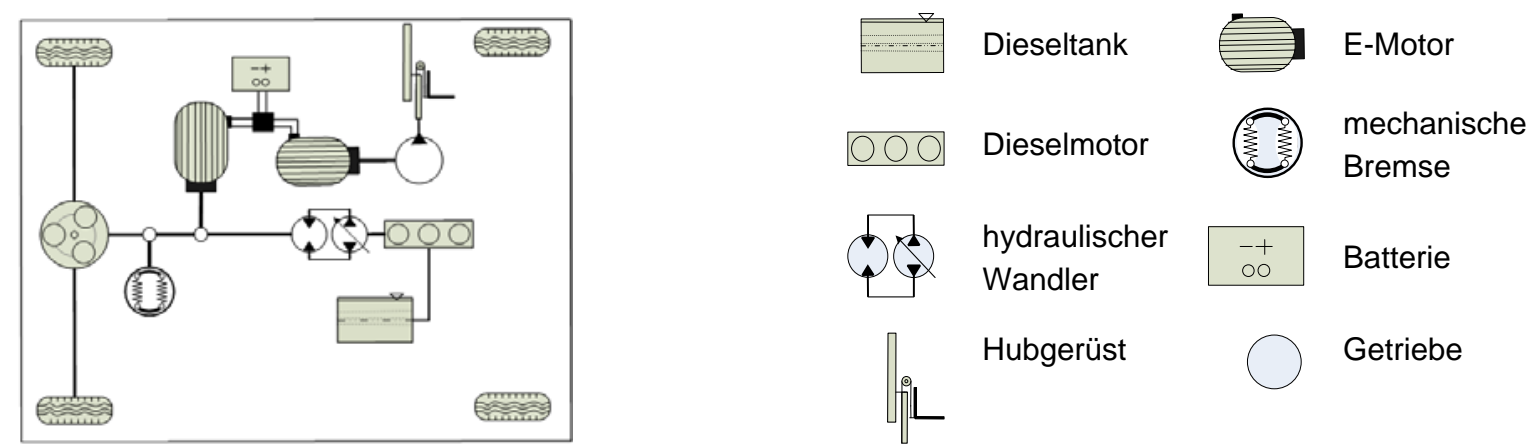

Abbildung 1: Schema des parallelen Hybridantriebes für einen Gegengewichtsstapler mit 2,5 t Tragkraft

Der im vorliegenden Beitrag als konkretes Beispiel dienende Hybridantrieb ist ein dieselelektrischer Parallelhybrid mit Momentenaddition für einen Gegengewichtsstapler mit einer Tragkraft von 2,5 t (Abbildung 1). Zwischen dem Dieselmotor und dem Antriebsrad ist ein hydrodynamischer Trilokwandler angeordnet, der die Funktion einer Schaltkupplung übernimmt und als Momentwandler dient. Parallel dazu ist über ein Getriebe ein Elektromotor angeordnet, der das Fahrzeug beschleunigen oder unter Rekuperation abbremsen kann. Während die Momente der Antriebselemente am Getriebe addiert werden sind die Drehzahlen fest mit der Raddrehzahl gekoppelt. Eine mechanische Bremse kommt darüber hinaus zum Einsatz, wenn die Bremskraft des Elektromotors nicht ausreicht oder die Batterie geladen ist. Die Hubhydraulik wird durch einen zweiten EMotor angetrieben. In Tabelle 1 sind die wesentlichen Daten das Antriebstranges zusammengefasst. Sie resultieren zum Teil aus Ergebnissen einer Optimierungsmethode für die Auslegung von Hybridantrieben für Gegengewichtsstapler, die am Lehrstuhl für Maschinenelemente und Technische Logistik entwickelt worden ist [St07].

Tabelle 1: Fahrzeugdaten

\begin{tabular}{|l|c|l|c|}
\hline Übersetzung E-Motor/Rad & $i_{E}=19,4$ & Übersetzung Wandler/Rad & $i_{W}=12,2$ \\
\hline Leistung des E-Motors & $P_{E}=24 \mathrm{~kW}$ & Leistung des Dieselmotors & $P_{D}=27,5 \mathrm{~kW}$ \\
\hline Fahrzeugmasse & $m_{F}=4290 \mathrm{~kg}$ & Radradius & $r_{R}=0,306 \mathrm{~m}$ \\
\hline Tragkraft & $m_{t}=2500 \mathrm{~kg}$ & Rollwiderstand & $f_{0}=0,016$ \\
\hline
\end{tabular}




\subsection{Betriebsstrategie}

Da jeder zusätzliche interne Motor des Antriebssystems einen zusätzlichen internen Betriebsfreiheitsgrad nach sich zieht, stellt sich gegenüber konventionellen Antrieben die Aufgabe der Auslegung der Betriebsstrategie. Dieser kommt die Funktion zu, die durch den Fahrer am Antriebsrad angeforderte Leistung auf die internen Motoren derart aufzuteilen, dass der Energieverbrauch minimiert wird. In nahezu allen Fällen wird diese Aufteilung durch die Nebenbedingung eingeschränkt, dass einer der Energiespeicher, sei es eine Batterie, ein Schwungrad, ein Druckspeicher oder ähnliches, eine begrenzte Kapazität aufweist. Insbesondere besitzt ein Parallelhybrid, der aus zwei mechanisch gekoppelten Motoren, die gleichzeitig auf das Antriebsrad wirken, besteht, einen Freiheitsgrad.

Die Betriebsstrategie hat zur Aufgabe, für ein gegebenes Geschwindigkeitsprofil bzw. Belastungsprofil einen zeitlichen Verlauf für die Leistungsteilung zu finden, so dass der Kraftstoffverbrauch minimiert wird. Da die Batterie im Gegensatz zum Kraftstofftank über einen begrenzten Energieinhalt verfügt und ein Vergleich zu konventionellen Antrieben gewährleitstet werden soll, wird darüber hinaus gefordert, dass der Ladezustand der Batterie zum Ende eines vorgegebenen Fahrmanövers genauso hoch ist wie zu Beginn. Es ergibt sich dadurch ein sogenanntes Optimalsteuerungsproblem der Form

$$
\begin{gathered}
\int_{0}^{t_{e}} \dot{m}_{K r}\left(u(t), \omega_{R}, M_{R}\right) d t=\min ! \\
N B: q(t=0)=q\left(t=t_{e}\right)
\end{gathered}
$$

Hierbei stellt $m_{K r}$ den aktuellen Kraftstoffverbrauch, $0 \leq q \leq 1$ den relativen Ladezustand der Batterie und $\omega_{R}$ und $M_{R}$ die Drehzahl bzw. das Moment des Antriebsrades dar. Der gesuchte Freiheitsgrad wird durch die Leistungsteilung $u(t)$ repräsentiert, die das Verhältnis der abgegebenen Leistung des Elektromotors zu der des Verbrennungsmotors repräsentiert.

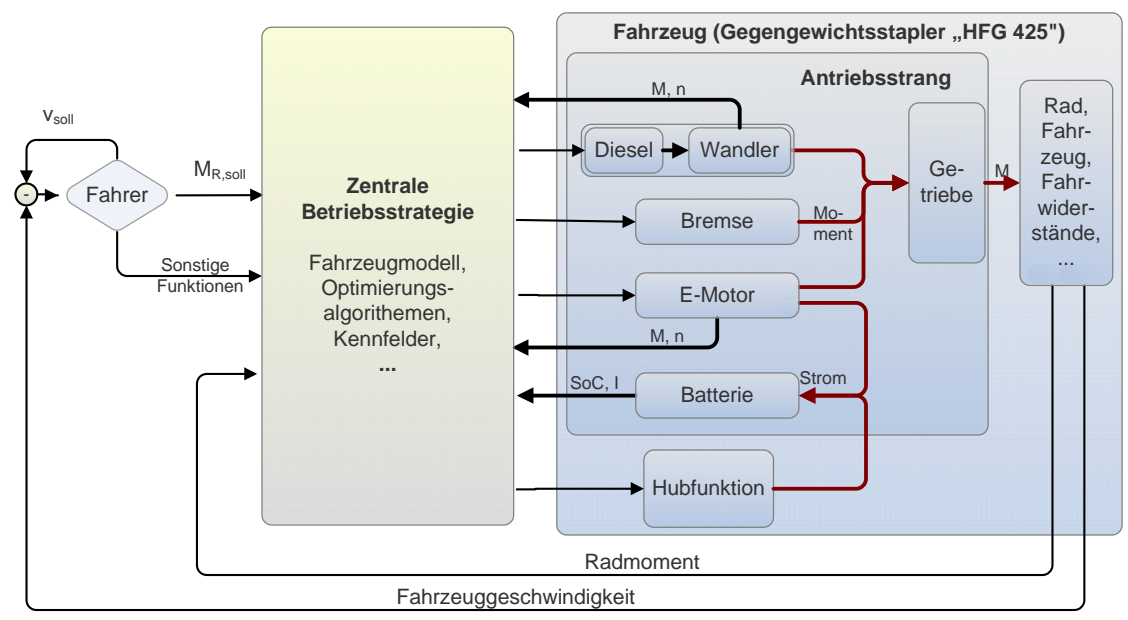

Abbildung 2: Struktur Paralleler Hybridantrieb mit zentraler Betriebsstrategie 
Im Allgemeinen ist (1) nicht praktikabel bzw. online implementierbar, da der gesuchte optimale zeitliche Verlauf der Leistungsteilung auch von zukünftigen Ereignissen abhängt. Das unter Anwendung der dynamischen Programmierung lösbare Optimierungsproblem kann jedoch als Benchmark für online implementierbare Betriebsstrategien dienen.

In den letzten Jahren ist eine äußerst große Vielfalt an Lösungsansätzen für online implementierbare Betriebsstrategien publiziert worden [GS07]. Allen gängigen Betriebsstrategien gemeinsam ist dabei ihr zentraler Charakter. Abbildung 2 zeigt die prinzipielle Struktur einer Betriebsstrategie für den oben vorgestellten dieselelektrischen Gegengewichtsstapler. Aufgrund des zusätzlichen Freiheitsgrades fungiert sie als Mittler zwischen dem Fahrer, der lediglich ein Fahrsignal gibt, und dem Antriebstrang, der zwei Fahrsignale erhält. Aufgrund von aktuellen Messdaten über den Systemzustand sowie detaillierter Fahrzeugmodelle wird die Entscheidung bezüglich des Leistungssplit fortlaufend neu getroffen. Die internen Fahrzeugmodelle können verschiedene Detailierungsgrade aufweisen. Je höher die Anforderungen an die Kraftstoffersparnis dabei sind, desto genauer müssen die Fahrzeugmodelle ausgearbeitet sein. Andererseits wächst dadurch der Änderungsaufwand des Fahrzeugmodels, wenn des Antriebssystems geändert werden.

\subsection{Benchmark der Betriebsstrategie - Dynamische Programmierung}

Die dynamische Programmierung hat sich in zahlreichen Fällen bewährt, um für ein gegebenes Fahrprofil den optimalen zeitlichen Verlauf der Leistungsabgabe der einzelnen Motoren eines Hybridantriebes zu bestimmen. Ihr wesentlicher Vorteil ist der im Vergleich zur direkten numerischen Lösung des nichtlinearen Problems stark reduzierter Rechenaufwand. Sie kann zwar nicht zur Echtzeitsteuerung des Systems angewandt werden, liefert aber hervorragende Ergebnisse, die im Vergleich mit online implementierbaren Betriebsstrategien als Benchmark dienen können.

Die dynamische Programmierung basiert auf dem Bellmaschen Optimalitätsprinzip und der daraus abgeleiteten Rekursionsformel [Be-57]. Es stellt sich dabei die Frage, wie man ein dynamisches System optimal von einem gegebenen Anfangszustand $z_{0}$ in einen gegebenen Endzustand $z_{n}$ in einer vorgegebenen endlichen Anzahl $n$ an Schritten $u_{k}$ mit $z_{k}=p_{k}\left(z_{k-1}, u_{k}\right)$ und $k=1, \ldots, n$ überführen kann. Gibt es eine optimale Folge von Zuständen $z_{0}, z_{1}^{*}, \ldots, z_{n-1}^{*}, z_{n}$, die die Kostenfunktion

$$
S^{*}\left(z_{0}, z_{n}\right)=\sum_{k=1}^{n} s_{k}\left(z_{k-1}^{*}, u_{k}^{*}\right) \rightarrow \min
$$

für den Systemübergang von $z_{0}$ nach $z_{n}$ minimiert und die Nebenbedingungen $z_{k} \in Z_{k}$ und $u_{k} \in U_{k}\left(z_{k-1}\right)$ erfüllt, dann ist entsprechend dem Optimalitätsprinzip von Bellmann jede Teilfolge $z_{\alpha}^{*}, z_{\alpha+1}^{*}, \ldots, z_{\beta-1}^{*}, z_{\beta}^{*}$ mit $0>\alpha>\beta \geq n$ bezüglich des Zustandsübergangs von $z_{\alpha-1}^{*}$ zu $z_{\beta}^{*}$ unter den gleichen Nebenbedingungen ebenfalls optimal. Unter Ausnutzung dieser Eigenschaft kann in absteigender Folge, ausgehend vom Zielzustand $n$, die optimale Folge mit Hilfe der Rekursionsformel

(C) 2010 Logistics Journal : Proceedings - ISSN 2192-9084 


$$
S^{*}\left(z_{k-1}^{*}, z_{n}\right)=\min _{u}\left\{s_{k}\left(z_{k-1}, u_{k}\right)+S^{*}\left(p_{k}\left(z_{k-1}, u_{k}\right), z_{n}\right) \mid u_{k} \in U_{k}\left(z_{k-1}\right)\right\}
$$

bestimmt werden.

Die Methode eignet sich sehr gut, um den optimalen Leistungssplitverlauf für einen gegebenen Hybridantrieb und ein gegebenes Fahrprofil zu ermitteln [KKJ+05]. $\mathrm{Zu}$ diesem Zweck werden die Zeit $t_{k}=k \frac{T}{n}$ mit $k=0, \ldots, n$ und der Endzeit $T$ und der Batterieladezustand $q_{l}=l \frac{Q}{m}$ mit $l=0, \ldots, m$ und dem maximalen Batterieladezustand $Q$ in hinreichend kleine Intervalle diskretisiert. Die Kostenfunktion wird dann durch den Kraftstoffverbrauch dargestellt, der Batterieladezustand entspricht dem Zustand $z$ des Systems und die Variable $u$ repräsentiert den Leistungssplit der beteiligten Motoren.

\section{Dezentrale Betriebsstrategie}

\subsection{Dezentraler Optimaler Regler - Zerlegung paralleler Komponenten}

Betrachtet man ein Optimierungsproblem der Form

$$
\begin{gathered}
F\left(x_{1}, \ldots, x_{n}\right)=\sum_{i=1}^{n} f_{i}\left(x_{i}\right) \rightarrow \min \\
N B: \quad g\left(x_{1}, \ldots, x_{n}\right)=\bar{x}-\sum_{i=1}^{n} x_{i}=0
\end{gathered}
$$

so lässt sich unter Verwendung Lagranger Multiplikatoren zeigen, dass in der Lösung des Optimierungsproblems gilt

$$
\frac{d f_{k}}{d x_{k}}=\frac{d f_{j}}{d x_{j}} \quad(k, j=1, \ldots, n)
$$

Dieser Zusammenhang kann zur Auslegung einer dezentralen optimalen Regelung für Hybridantriebe genutzt werden, wenn man die abgegebene Leistung der einzelnen Motoren des Antriebsystems $P_{m, i}$ mit den Variablen $x_{i}$ und die vom Betriebspunkt abhängige Verlustleistung $P_{V, i}$ der einzelnen Motoren mit $f_{i}$ gleichsetzt. In diesem Fall stellt die in der Nebenbedingung enthaltene Konstante $\bar{x}$ die durch den Fahrer vorgegebene Sollleistung am Rad dar. Alternativ zur Besetzung der Funktionen $f_{i}$ mit den Motorverlustleistungen können diese auch mit den jeweiligen Eingangsleistungen besetzt werden, da die Minimierung der Verlustleistung bei vorgegebener Ausgangsleitung äquivalent zur Minimierung der Eingangsleistung ist. Unter Anlehnung an den Zusammenhang (5) wird dann der Korrekturterm

$$
c=\left(\frac{\partial P_{e}}{\partial P_{m}}\right)_{\kappa}
$$

definiert [FB10]. Die Ableitung der Eingangsleistung $P_{e}$ eines Motors nach dessen mechanischer Ausgangleistung $P_{m}$ wird dabei unter "festhalten“ aller festen, von außen vorgegebenen, Zustandsvariablen $\kappa$ gelbildet. Für Motoren kommen dabei in (c) 2010 Logistics Journal : Proceedings - ISSN 2192-9084 
der Regel die Rotationsgeschwindigkeit $\omega$ und das Motormoment $M$ in Frage, da diese beiden Zustandsvariablen über den Wirkungsgrad $\eta(\omega, M)=P_{m} / P_{e}$ maßgeblich den Zusammenhang von Eingangsleistung und Ausgangsleistung bestimmen. Insbesondere ist im Fall eines wie oben beschriebenen Parallelhybridantriebes mit Momentenaddition das Moment die freie Variable, da die Rotationsgeschwindigkeit durch die Raddrehzahl fest vorgegeben ist. Unter festhalten der Rotationsgeschwindigkeit führt (6) damit zu

$$
c=\eta^{-1}+M \frac{\partial \eta^{-1}}{\partial M}
$$

und somit zu einer Definition eines Korrektorterms $c$, der verwendet werden kann, um den Hybridantrieb dezentral und dennoch optimal zu regeln.

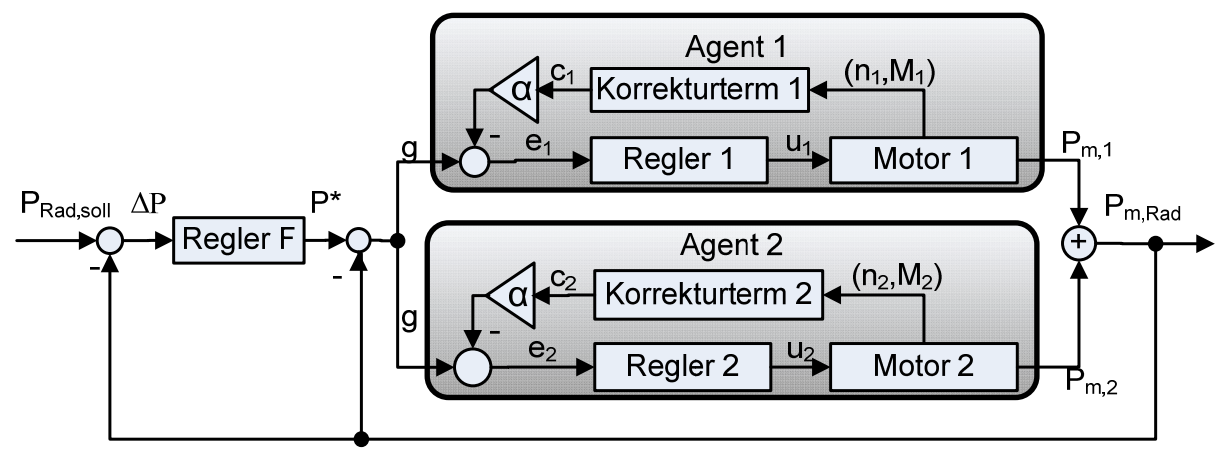

Abbildung 3: Reglerstruktur der dezentralen optimalen Regelung

Die konkrete Umsetzung dieser Vorgehensweise wird in Abbildung 3 deutlich. Dargestellt ist schematisch ein Parallelhybrid mit den Motoren 1 und 2, deren mechanische Ausgangsleistung sich über ein Getriebe zur Radausgangsleistung addiert. Über den Gesamtfahrzeugregler F wird die tatsächliche Radleistung auf die vom Fahrer vorgegebene Sollradleistung eingeregelt. Dies und ein weiterer Vergleich des Reglerausganges von $\mathrm{F}$ mit der Radleistung ist trotz der beiden lokalen Motorregler 1 und 2 notwendig, da der Eingang $g$ aufgrund der Korrektorterme eine bleibende Regelabweichung aufweist. Bei adäquater Auslegung der beteiligten Einzelregler verschwindet sowohl die Leistungsdifferenz $\Delta P$, wodurch die Nebenbedingung in (4) des oben vorgestellten Optimierungsproblems erfüllt ist, als auch die Eingänge der beiden lokalen Motorregler $e_{1}$ und $e_{2}$. Daraus lässt sich aber wiederum folgern, dass die beiden Korrektorterme $c_{1}$ und $c_{2}$ gleich groß sind, die Bedingung (5) dadurch erfüllt und die Summe der Eingangsleistungen respektive der dissipierten Leistungen der beiden Motoren dementsprechend minimiert ist.

\subsection{Zerlegung serieller Komponenten - Batterie und Elektromotor}

Der dezentrale Regler aus dem oberen Abschnitt berücksichtigt das Batterielademanagement noch nicht. Um dieses zu implementieren, kann zunächst davon ausgegangen werden, dass einer der Motorblöcke aus Abbildung 3 sowohl den Elektromotor selbst als auch die Batterie umfasst. Die zugehörige Modelvorstellung ist in Abbildung 4 dargestellt. Die dezentrale Implementierung des 
Batterielademanagements erfolgt nun in zwei Schritten. Zunächst wird der kombinierte Korrektorterm in zwei voneinander unabhängige Korrektorterme jeweils für den Motor und die Batterie zerlegt. Anschließend wird ein vom Ladezustand abhängiger virtueller Batteriewirkungsgrad eingeführt, über den das Systemverhalten derart gesteuert werden kann, dass der Batterieladezustand jederzeit innerhalb vorgegebener Grenzen bleibt.

Die Batterie (Abbildung 4) ist durch einen reinen chemischen Energiespeicher und einen Energiewandler modelliert, der die chemisch gespeicherte Energie unter Verlust in elektrische Energie wandelt und umgekehrt. Darüber hinaus ist eine Abzweigung elektrischer Leistung modelliert, die durch Nebenverbraucher wie z.B. die Hubfunktion bedingt ist. Der Korrektorterm für diesen kombinierten Block aus Batterie und E-Motor, der die Summe der dissipierten Leistungen berücksichtigt, berechnet sich aus der Ableitung der chemischen Leistung der Batterie nach der mechanischen Leistung des E-Motors.

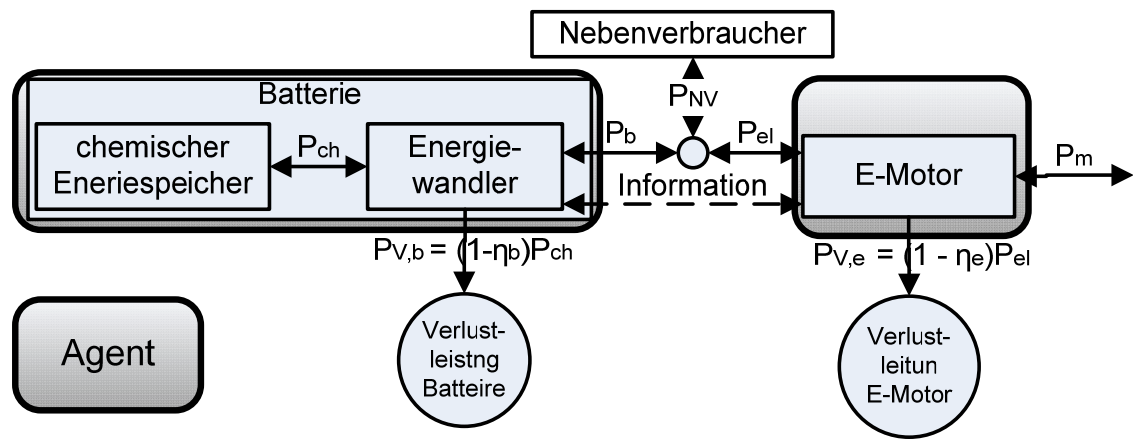

Abbildung 4: Batterie und E-Motor

Bezüglich der Zerlegung des Gesamtkorrekturterms $c_{m / b}=\left(\partial P_{c h} / \partial P_{m}\right)_{\kappa}$ lässt sich zeigen [Fr-10], dass sich dieser unabhängig von der Leistung der Nebenverbraucher $P_{N V}$ als Produkt der beiden Einzelkorrektorterme für die Batterie $c_{b}=\left(\partial P_{c h} / \partial P_{b}\right)_{\kappa}$ und den Motor $c_{m}=\left(\partial P_{e l} / \partial P_{m}\right)_{\kappa}$ darstellen lässt. Unter der vereinfachenden Annahme, dass die Spannung der Batterie konstant ist und unter der Annahme ,Hybridantrieb mit Momentaddition‘ ergibt sich mit dem Batteriestrom $I_{b}$ weiterhin

$$
c_{m / b}=c_{m} \cdot c_{b}=\left(\eta_{e}^{-1}+M \frac{\partial \eta_{e}^{-1}}{\partial M}\right) \cdot\left(\eta_{b}^{-1}+I_{b} \frac{\partial \eta_{b}^{-1}}{\partial I_{b}}\right)
$$

Damit lässt sich die Verlustleistung der Batterie zunächst unabhängig von der des Elektromotors betrachten.

\subsection{Dezentrales Batteriemanagement}

Das für den zweiten Schritt angekündigte Batteriemanagement lässt sich nun zunächst als These durch

$$
\eta_{b}=\eta_{b, r}\left(I_{b}\right) \cdot \eta_{b, v}\left(q_{b}\right)
$$


das Produkt des vom Batteriestrom abhängigen realen Wirkungsgrad $\eta_{b, r}$ und einem vom Batterieladezustand $q_{b}$ abhängigen virtuellen Wirkungsrad $\eta_{b, v}$ darstellen. Zur Darstellung des virtuellen Batteriewirkungsgrades kann die Eigenschaft des Systems ausgenutzt werden, zu jeder Zeit gegen den Betriebspunkt mit dem geringsten Energieverlust zu streben. Da Aufgrund von (8) nun die Summe der Verlustleistungen des Verbrennungsmotors, des E-Motors und der Batterie minimiert wird, reicht es aus, dem System einen hohen Batteriewirkungsgrad für gewünschte Batteriezustände und umgekehrt eine besonders schlechten Batteriewirkungsgrad für ungewünschte Batteriezustände vorzugeben. Dazu können zunächst sechs verschiedene Batteriezustände definiert und bewertet werden (Tabelle 2). Jedem Batteriezustand wird dann eine virtuelle Verlustleistung zugeordnet, die gegen negativ unendlich für gewünschte Zustände und positiv unendlich für ungewünschte Zustände strebt.

Tabelle 2: Batteriezustände und deren Bewertung

\begin{tabular}{|c|c|c|c|c|}
\hline & $\begin{array}{c}\text { Batterieladezustand } \\
\boldsymbol{q}=\mathbf{0 . . 1}\end{array}$ & $\begin{array}{c}\text { relative Batterieleistung } \\
\boldsymbol{\rho}=\boldsymbol{P}_{\boldsymbol{b}} / \boldsymbol{P}_{\boldsymbol{b}, \boldsymbol{m a x}} \mid\end{array}$ & Bewertung & $\begin{array}{c}\text { virtu. Verlustleistung } \\
\boldsymbol{\gamma}=\boldsymbol{P}_{\boldsymbol{V}, \boldsymbol{b}} / \boldsymbol{P}_{\boldsymbol{b}, \boldsymbol{m a x}} \mid\end{array}$ \\
\hline 1 & $q=1$ & $\rho=1$ & gewünscht & $\gamma \rightarrow-\infty$ \\
\hline 2 & $q=1$ & $\rho=-1$ & ungewünscht & $\gamma \rightarrow \infty$ \\
\hline 3 & $q=0$ & $\rho=1$ & ungewünscht & $\gamma \rightarrow \infty$ \\
\hline 4 & $q=0$ & $\rho=-1$ & gewünscht & $\gamma \rightarrow-\infty$ \\
\hline 5 & $q=0.5$ & $\rho=-1 . .1$ & neutral & $\gamma=0$ \\
\hline 6 & $q=0 . .1$ & $\rho=0$ & neutral & $\gamma=0$ \\
\hline
\end{tabular}

Für die Verlustleistung der Batterie kann dann der Zusammenhang $\gamma=\rho(0.5-q) /[q(1-q)]$ dienen, der auf eine batterieleistungsunabhängige Form für den virtuellen Wirkungsgrad führt.

$$
\eta_{b, v}(q)=\left(q-q^{2}\right)\left(0,5-q^{2}\right)^{-1}
$$

Im Gegensatz zu der Zerlegung des Antriebsstranges in die Einzelkomponenten, bei der ein lokales und momentanes Energieoptimum sichergestellt ist, ist die Optimalität über einem Fahrprofil bei diesem Ansatz nicht sichergestellt. Die Güte des gewählten Ansatzes kann aber im Vergleich mit einem durch die dynamische Programmierung ermittelten optimalen Fahrprofil bewertet werden. 


\subsection{Zusammenfassung}

Die Regelung des in Abbildung 1 dargestellten Hybridantriebes mit der so entwickelten Methode bedarf der drei Einzelregler für die Bremse, den Dieselmotor und den Elektromotor. Die Hubfunktion ist als Nebenverbraucher (siehe Abbildung 4) bereits intergiert. Basierend auf den obigen Ergebnissen werden dann die Korrektorterme für die einzelnen Regler

$$
\begin{gathered}
c_{d / t}=\eta_{d / t}^{-1}+M_{W} \frac{\partial \eta_{d / t}^{-1}}{\partial M_{W}} \\
c_{m / b}=c_{m} \cdot c_{b} \cdot \eta_{b, v}^{-1}
\end{gathered}
$$

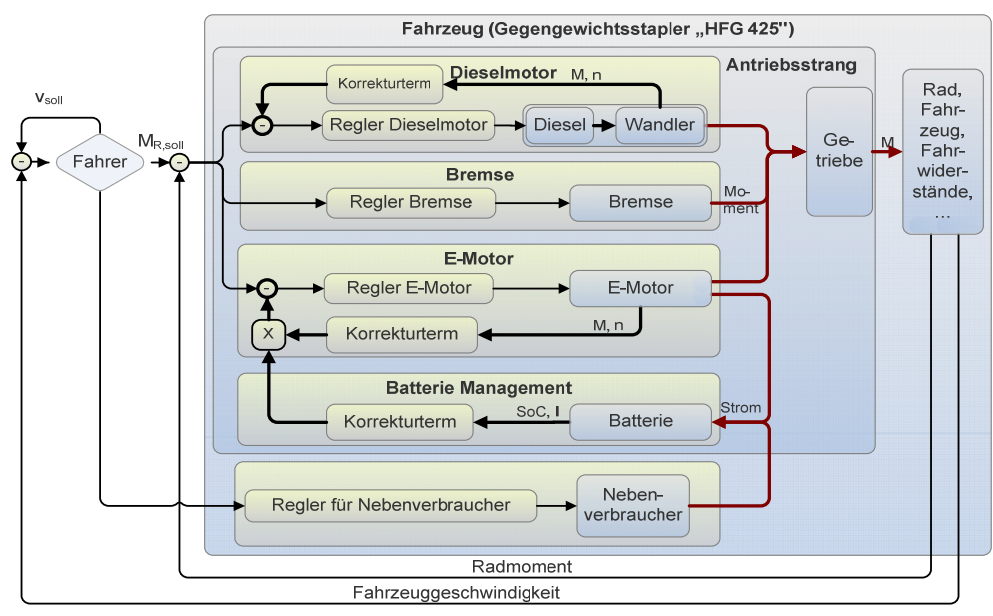

Abbildung 5: Gesamtfahrzeugregler der dezentralen optimalen Reglerstruktur

Der Korrektorterm für die Dieselmotor/Trilokwandler Einheit $c_{d / t}$ wird mittels des kombinierten Wirkungsgrades $\eta_{d / t}$ und des Ausgangsmomentes des Trilokwandlers $M_{W}$ gebildet. Da die Eingangsleistung der Bremse zu jedem Zeitpunkt null ist, ist auch die Ableitung der Eingangsleistung und dementsprechend der

Bremskorrektorterm zu jedem Zeitpunkt null. Die so erhaltene dezentrale und optimale Fahrzeugreglerstruktur ist in Abbildung 5 dargestellt.

\section{$4 \quad$ Vergleich der dezentralen Regelung mit dem optimalen Ergebnis}

Im Folgenden wird der zeitliche Verlauf der Leistungsteilung der beiden Motoren eines parallelen Hybridantriebes, der sich durch die vorgestellte dezentrale optimale Betriebsstrategie einstellt, mit der optimalen Lösung, die mithilfe der dynamischen Programmierung ermittelt worden ist, verglichen. Der zugehörige Geschwindigkeitsverlauf des Fahrzeugs ist dem standardisierten Arbeitsspiel für Flurförderzeuge der Norm VDI 2198 [VD02] entnommen.

\subsection{Fahrprofil}

Das Arbeitsspiel setzt sich aus einer vorgegebenen Fahrtstrecke zwischen zwei $30 \mathrm{~m}$ voneinander entfernten Punkten $A$ und $B$ zuzüglich eines Wendevorganges und einem Hubvorgang mit Nennlast im Stillstand zusammen und wird 120-mal in einer Stunde durchgeführt. Da die benötigte Leistung für den Hubvorgang im Stillstand (c) 2010 Logistics Journal : Proceedings - ISSN 2192-9084 
aufgrund der Antriebsstrangarchitektur (Abbildung 1) lediglich der Batterie entspringt, befindet sich das Fahrzeug nur während der Fahrt im hybriden Betriebszustand. Die Simulation bezieht sich daher nur auf die ersten 16,7 s des Arbeitsspiels in denen sich das Fahrzeug bewegt. Die Batterie muss in dieser Zeit um genau den Betrag zusätzlich geladen werden, der für den Hubvorgang benötigt wird. Voruntersuchungen mit einem konventionellen Stapler auf Experiment- und Simulationsbasis haben ergeben, dass bei einer Nettohublast von 2,5 $\mathrm{t}$ auf eine Hubhöhe von $2 \mathrm{~m}$ inklusive Energierückgewinnung beim Senken ca. $61 \mathrm{~kJ}$ aufgebracht werden müssen. Dies ist gleichzusetzen mit einer zusätzlichen

Batteriebelastung von etwa 3,65 kW während der simulierten 16,7 s Fahrtzeit. Am Ende des Fahrprofils, nach dem abgeschlossenen Hubvorgang, ist dann der Batterieladezustand genauso hoch wie zu Beginn. Um Energie zu sparen sei in den Antriebsstrang eine Start-Stopp-Automatik implementiert, sodass der Verbrennungsmotor abgeschaltet ist während das Fahrzeug steht. Da die
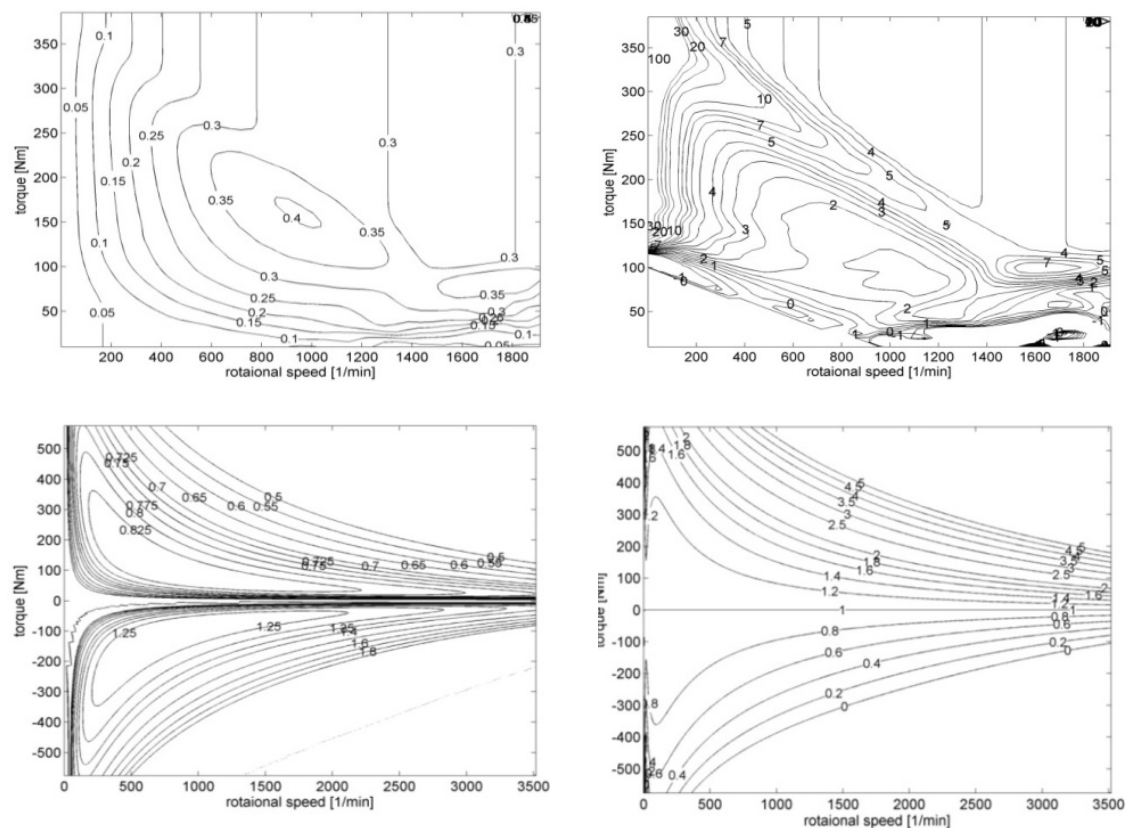

Abbildung 6: Oben links: Wirkungsgradkennfeld VKM/Wandler; oben rechts: Korrektorkennfeld VKM/Wandler; unten links:

Wirkungsgradkennfeld E-Motor; unten rechts: Korrektorkennfeld E-Motor
Fahrtrichtung bei Staplern lediglich durch ein internes Wendegetriebe vorgegeben wird ist es für den Antriebsstrang irrelevant, ob der Stapler vorwärts oder rückwärts fährt. Zur Vereinfachung wurde die Rückwärtsbewegung des Staplers im gewählten Fahrprofil nach VDI 2198 durch eine Vorwärtsfahrt ersetzt.

\subsection{Simulationsmodel}

Der in 2.1 vorgestellte parallele Hybridantrieb dient als Basis. Dazu ist ein dynamisches Simulationsmodell des Fahrzeugs in Matlab-Simulink implementiert worden. Für die Einzelkomponenten Verbrennungsmotor, Trilokwandler, Getriebe, Hubhydraulik, Bremse und Fahrzeug standen bereits validierte Teilmodelle in Simulink zur Verfügung, die einem Simulationsmodell eines Gegengewichtsstaplers mit konventionellem Antrieb entnommen wurden. Das Modell für die Batterie basiert auf einer Reihenschaltung eines konstanten ohmschen Widerstandes und einer konstanten Spannungsquelle von $80 \mathrm{~V}$ sowie eines konstanten 
Batterieladewirkungsgrades von $90 \%$. Der Elektromotor ist mithilfe eines Wirkungsgradkennfeldes für die Motorverluste modelliert.

Die Verluste der zum Motor gehörenden Leistungselektronik sind in das Motorkennfeld integriert. Zusammen mit dem kombinierten Wirkungsgradkennfeld von Verbrennungsmotor und Trilokwandler bilden die beiden Antriebseinheiten die Hauptverlustquellen des Antriebsstranges. Die beiden Wirkungsgradkennfelder sind zusammen mit den entsprechend Gleichung (7) ermittelten Korrektorkennfelder in Abbildung 6 dargestellt.
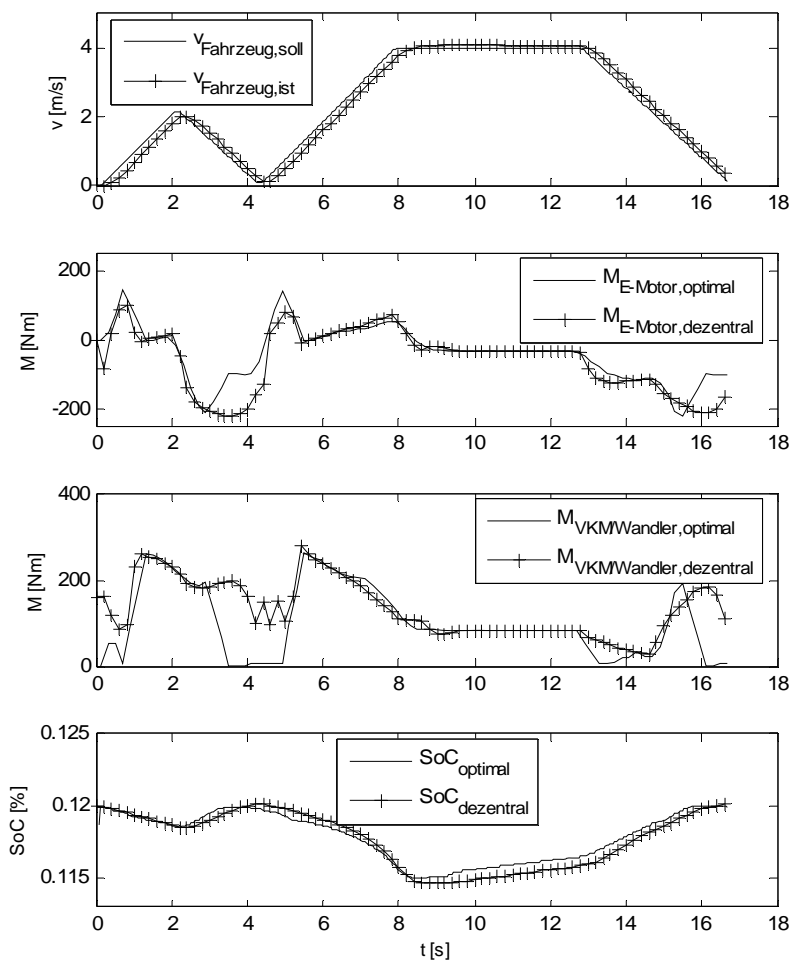

Abbildung 7: Vergleich der Ergebnisse der dynamischen Programmierung mit der dezentralen Regelung (oben: Geschwindigkeitsprofil soll und ist; 2. v. oben: Momentverlauf des Elektromotors; 3. v. oben: Momentverlauf des Trilokwandlers; unten: Batterieladezustand)

Die Reglerstruktur des Fahrzeugs ist so gewählt wie in Abbildung 5 dargestellt. Die Korrektorterme werden entsprechend den Gleichungen (11) und (12) bestimmt. Dabei können die Werte für die beiden Motoren und die Batterie mit Hilfe von Kennfeldern implementiert werden. Die Regler für die Motoren und die Bremse werden durch PI-Regler mit drehzahlabhängiger Drehmomentbegrenzung dargestellt. In der Regelung des Verbrennungs-motors kommt darüber hinaus eine FeedbackLinearisierung für das kombinierte Übertragungsverhalten von Motor und Trilokwandler zum Einsatz. Dadurch können die einzelnen Regler im dezentralen Sinne unabhängig voneinander ausgelegt werden. Der Fahrer ist über eine Vorsteuerung des Radmomentes und einen PID-Regler realisiert. Für die Ermittlung des optimalen Fahrprofils mit Hilfe der dynamischen Programmierung wurde das Simulationsmodell des Hybridstaplers stark vereinfacht als statisches Modell [Gu07] und mit einem verschwindenden Batterieinnenwiderstand implementiert. Die Rechenzeit konnte damit trotz einer relativ feinen Diskretisierung von $\Delta t=0,05 \mathrm{~s}$ und $\Delta q=5 \cdot 10^{-5}$ auf etwa 4 Stunden mit einem handelsüblichen PC gesenkt werden.

\subsection{Simulationsergebnisse}

Abbildung 7 zeigt die Ergebnisse der dynamischen Programmierung mit denen der dezentralen Regelung im Vergleich. Es zeigt sich eine äußerst hohe Übereinstimmung zwischen der optimalen Lösung mit dem für dieses Fahrmanöver geringsten Kraftstoffverbrauch und dem Ergebnis der dezentralen Regelung. 
Insbesondere bei hohen Geschwindigkeiten zwischen der 5. und der 13. Sekunde ist die Leistungsteilung nahezu identisch. Demgegenüber steht die äußerst simple und hochflexible Reglerauslegung entsprechend Abbildung 5. Trotz der guten Ergebnisse ist hier kein zusätzliches Fahrzeugmodel notwendig. Die Ergebnisse untermauern damit das Potential der dezentralen optimalen Regelung einerseits und andererseits lässt sich der gewählte Ansatz für das dezentrale Batterielademanagement entsprechend (10) begründen.

\section{$5 \quad$ Zusammenfassung}

Im vorliegenden Beitrag konnte gezeigt werden, wie die Regelung und das Batterielademanagement für einen Hybridantrieb dezentral ausgelegt werden kann. Dabei fließen bei der Auslegung einer Regelungskomponente für einen Motor des Antriebs keinerlei Informationen über den Rest des Systems wie Getriebe, Fahrzeug, Batterieeigenschaften oder weitere Motoren ein. Weiterhin konnte gezeigt werden wie es trotz des dezentralen Charakters der Regelung möglich ist, dass das System zu jedem Zeitpunkt gegen den internen Zustand mit dem globalen Wirkungsgradoptimum konvergiert. Schließlich konnte gezeigt werden, wie mit Hilfe eines dezentralen Ansatzes für das Batterielademanagement auch ein sehr guter Leistungssplitverlauf für ein gegebenes Fahrprofil implementiert werden kann, sodass der Batterieladezustand stets zwischen zwei vorgegebenen Grenzen gehalten wird. Da das Grundproblem (4) sehr allgemein gehalten ist, lässt sich der Ansatz nicht nur auf weitaus komplexere Antriebsstrukturen anwenden, sondern auch auf andere Regelungsaufgaben für verteilte Netzwerke mit internen Freiheitsgraden.

\section{Literatur}

[Be57] Bellmann, R. E.: Dynamic Programming. Princeton: Princeton University Press, 1957.

[FB10] Frenkel, A.; Bruns, R.: Decentralized optimal control strategy for parallel hybrid electric vehicles. VDI Berichte 2081, 2010.

[GS07] Guzzella, L., Sciarretta, A.: Vehicle Propulsion Systems - Introduction to Modeling and Optimization. Berlin: Springer, 2007.

[KKJ+ 05] Koot, M. W. T.; Kessels, J. T. B. A.; De Jager, A. G.; Heemels, W. P. M. $\mathrm{H}$.; Van den Bosch, P. P. J.; Steinbuch, M.: Energy management strategies for vehicular power systems. IEEE Transactions on Vehicular Technology, 54 (3):771-782, 2005.

[St07] Steiger, S.: Entwicklung eines innovativen hybriden Antriebssystems für Flurförderzeuge. Hamburg: HSU, Dissertation, 2007.

[UN03] Reg. 83 - Rev.3: Emission of pollutants according to engine fuel requirements; by UN Economic Commission for Europe, 2003.

[VD02] VDI 2198: Typenblätter für Flurförderzeuge. Düsseldorf: Verein Deutscher Ingenieure, 2002. 